\section{D-Printable Platform for High-Throughput Small-Animal Imaging}

TO THE EDITOR: There is considerable interest in preclinical imaging techniques to increase throughput and reduce costs, as evidenced by recent and former publications in the field (1-3). We write this letter to share a design for a multiple-mouse imaging platform that can be produced on a 3-dimensional (3D) printer and has been in use at our institution and several others for smallanimal PET/CT. We feel this design would likewise benefit the community, particularly at facilities that encourage do-it-yourself approaches. Our design is inexpensively manufactured with 3D printers, which are increasingly accessible at many institutions. Because of the compact size of our design, it is easy to integrate with a wide variety of scanner models and apply to various imaging configurations. We have provided the design files as supplemental materials (available at http://jnm.snmjournals.org), along with a short video demonstrating assembly of the components. We highly encourage the reader to watch the video, as it thoroughly details the ease of construction and utility of our design.
The use of this design for murine PET/CT imaging was conducted under a protocol approved by the Institutional Animal Care and Use Committee and Research Animal Resource Center of Memorial Sloan Kettering Cancer Center (MSKCC). The design (Fig. 1) was generated in the open-source computer-aided modeling software Blender, making use of Boolean operations on geometric primitives and a small amount of basic mesh manipulation. Several inexpensive, commercially available components were used for connection to an anesthesia system:

- 4 barbed unified-national-fine (UNF)-thread male pipe adapters, quarter-inch $(6.35 \mathrm{~mm}), 28$ threads per inch, having an eighth-inch (3.175 mm) internal diameter (Cole Parmer model 31501-54).

- 4 female Luer bulkheads, quarter-inch $(6.35 \mathrm{~mm}), 28$ threads per inch, with an eighth-inch $(3.175 \mathrm{~mm})$ internal diameter hose barb adaptor (Cole Parmer model 45508-34).

- 4 1-way stopcocks with female Luer inlet, male Luer lock outlet (Cole Parmer model 30600-00).

- 4 male Luer locks with lock rings and an eighth-inch (3.175 mm) internal diameter hose barb adaptor (Cole Parmer model 45504-04).

- 3 barbed Y-connectors having an eighth-inch $(3.175 \mathrm{~mm})$ internal diameter (Cole Parmer model 30703-92).

- Tygon (Saint-Gobain Corp.) tubing having a 3.1-mm internal diameter (Cole Parmer model 06440-16).

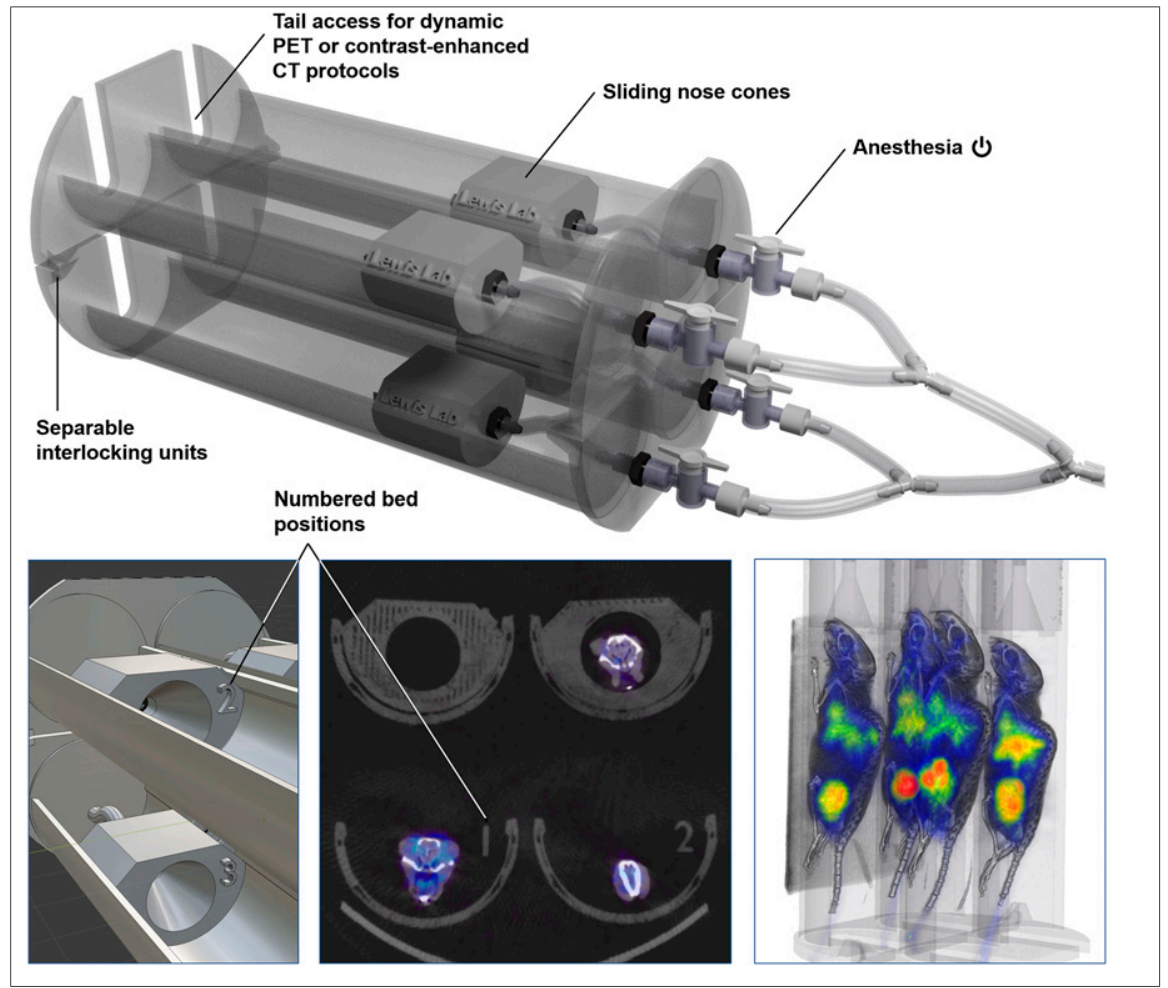

FIGURE 1. (Top) Rendering of 3D-printable multiple-mouse imaging platform and design features. (Bottom left and middle) Platform and axial PET/CT images acquired using it. (Bottom right) Overlay of PET and CT volume-rendered projections with bed geometry within 3D Slicer software.

COPYRIGHT @ 2020 by the Society of Nuclear Medicine and Molecular Imaging.
After 3D printing, assembly requires approximately only $5 \mathrm{~min}$ using the following basic tools: a drill, a quarter-inch (6.35 mm) 28 threads per inch UNF tap (Grainger model 427R24), an 8-mm openended wrench (Grainger model 36T946), and scissors or a utility knife.

The design has been tested for compatibility with the Siemens Inveon and Concorde microPET Focus 120 scanners, and care was taken to ensure that the design would be easily modifiable should it need to be shortened, lengthened, or otherwise adjusted for compatibility with other scanners or animal sizes or to accommodate an experimental apparatus or other desired feature.

We encourage the reader to refer to previous detailed discussions (1-3) of the resolution and image quality obtainable with standard radionuclides $\left(\right.$ e.g., $\left.{ }^{18} \mathrm{~F},{ }^{11} \mathrm{C}\right)$ in simultaneous multiple-animal imaging. We stress that use of this or similar designs for simultaneous multiple-animal PET imaging demands that appropriate acceptance testing measures for quality assurance be conducted, especially when quantitative images are required. In addition to the standard National Electrical Manufacturers Association NU-4 2008 (4) tests for image quality, we recommend that some tests also be assessed at each bed position, including uniformity, spatial resolution, and activity recovery or spillover. As in the present 
design, the bed positions are offset from the center of the field of view (where spatial resolution is maximal). The spatial resolution should be known or evaluated to a radial extent at least as large as the radius of a circle circumscribing the 4 bed positions $(\sim 4 \mathrm{~cm})$. Additionally, because simultaneously imaging multiple mice typically involves elevated activities within the scanner field of view, it is critical to consider the scanner count rate performance in order to ensure the accuracy of detector dead-time corrections. Attention to scanner count rate performance is particularly important for nonstandard radionuclides, as there will be two considerations in addition to the aforementioned ones. First, many nonstandard positron emitters emit concomitant $\gamma$-rays, which may significantly contribute to dead-time counting losses, especially with the elevated activities within the field of view associated with simultaneously imaging multiple mice. Second, prompt $\gamma$-coincidences, which occur for radionuclides with $\gamma$-emissions within-or downscattering into- the positron annihilation photon energy window (e.g., ${ }^{86} \mathrm{Y},{ }^{124} \mathrm{I}$ ), are amplified when multiple mice are within the scanner field of view. We note that preclinical PET imaging workflows for mice often neglect corrections for attenuation and scatter because of their relatively minor impact for small animals such as mice; however, we recommend these corrections for multiple-mouse imaging because of the increased quantity of attenuating material and the increased likelihood of scatter. Finally, we note that there are no provisions included in our design for animal monitoring or body temperature maintenance, which may be required in, for example, ${ }^{18} \mathrm{~F}$-FDG imaging or extended scanning periods. Provisions for monitoring or temperature control may be added by users but should be evaluated by their Institutional Animal Care and Use Committee before application.

The use of multiple-animal imaging protocols at our institution has greatly streamlined many imaging studies while reducing cost. We greatly value and encourage recent efforts, both commercially aligned and community-contributed, toward development of imaging solutions to increase throughput while maintaining quantitative accuracy.

\section{DISCLOSURE}

Funding was received from the Mr. William H. and Mrs. Alice Goodwin and the Commonwealth Foundation for Cancer Research, the Center for Experimental Therapeutics of MSKCC, and National Institutes of Health grant R35 CA232130 (Jason Lewis); the Ruth L. Kirschstein fellowship (NIH F32 EB025050) (Lukas Carter); and the Tow fellowship via the Center for Molecular Imaging and Nanotechnology at MSKCC (Kelly Henry). No other potential conflict of interest relevant to this letter was reported.

\section{REFERENCES}

1. Greenwood HE, Nyitrai Z, Mocsai G, Hobor S, Witney TH. High-throughput PET/CT imaging using a multiple-mouse imaging system. J Nucl Med. 2020;61: 292-297.

2. Reilhac A, Boisson F, Wimberley C, et al. Simultaneous scanning of two mice in a small-animal PET scanner: a simulation-based assessment of the signal degradation. Phys Med Biol. 2016;61:1371-1388.

3. Yagi M, Arentsen L, Shanley RM, Hui SK. High-throughput multiple-mouse imaging with micro-PET/CT for whole-skeleton assessment. Phys Med. 2014;30:849-853.

4. NEMA Standard Publication NU 4-2008: Performance Measurements of Small Animal Positron Emission Tomographs. Arlington, VA: National Electrical Manufacturers Association; 2008.

Lukas M. Carter Kelly E. Henry

\author{
Andre Platzman \\ Jason S. Lewis* \\ *Memorial Sloan-Kettering Cancer Center \\ 1275 York Ave. \\ New York, NY 10065 \\ E-mail: lewisj2@mskcc.org
}

Published online Apr. 13, 2020.

DOI: 10.2967/jnumed.119.240457

\section{${ }^{11}$ C-(+)-PHNO Trapping Reversibility for Quantitative PET Imaging of $\beta$-Cell Mass in Patients with Type 1 Diabetes}

TO THE EDITOR: For efficiently differentiating the pancreas uptake of the dopamine $\mathrm{D}_{2} / \mathrm{D}_{3}$-receptor agonist ${ }^{11} \mathrm{C}-(+)$-PHNO $(3,4,4 a, 5,6,10 b$-hexahydro- $2 H$-naphtho[1,2-b][1,4]oxazin-9-ol) between healthy controls and patients with type 1 diabetes mellitus (T1DMs), Bini et al. recently compared different methods of quantitative PET imaging. These methods involved tissuecompartment model analyses providing the tracer distribution volume, as well as reference-region approaches, which did and did not require arterial sampling, respectively (1). Quantitative parameters were also correlated to clinically relevant measures of $\beta$-cellmass function such as $\mathrm{C}$-peptide, proinsulin, age at diagnosis, and disease duration. The authors reported a reduction in the 20- to 30min pancreas-to-spleen SUV ratio of $36.2 \%$ between healthy controls and T1DMs $(P=0.03)$ and concluded that this ratio could be used to differentiate $\beta$-cell mass in healthy controls and T1DMs.

We assume that the study of Bini et al. did not fully take into account the trapping reversibility of ${ }^{11} \mathrm{C}-(+)-\mathrm{PHNO}$ in the pancreas of both healthy controls and T1DMs - a characteristic that could, in addition, be helpful for assessing $\beta$-cell mass using data acquired beyond $30 \mathrm{~min}$ after injection. Trapping reversibility is evidenced in Figure 3 of the paper, in which representative decaycorrected time-activity curves of the pancreas (and spleen) acquired over 120 min clearly do not reach a plateau at late imaging $(1,2)$.

First, let us note that trapping reversibility may impair the use of distribution volume, that is, the equilibrium ratio of tissue concentration to plasma concentration, when this ratio is assessed at any time after injection, unlike under irreversible trapping condition (3).

Second, Bini et al. acknowledged that the 1-tissue-compartment model does not fit the data for times of more than $60 \mathrm{~min}$ (Fig. 3) (1). In this connection, we have recently indicated that a previously published method can then be applied to any tracer for assessing its release rate constant from tissue back to blood at late imaging, that is, when the part of free tracer in blood and interstitial volume plus, possibly, the part of radiolabeled metabolites have become negligible in the tissue time-activity curve (4). Comparison between arterial input function and pancreas SUV (Figs. 1 and 3, respectively) shows that this part is less than $2 \%$ at $30 \mathrm{~min}$ after injection, thus allowing the fitting of the pancreas (decaycorrected) time-activity curve beyond $30 \mathrm{~min}$ after injection with a monoexponentially decaying function (GraphPad Prism software, version 5.00), writing $y=37.54 \times \exp (-0.02621 \times t)$, where 0.02621 $\min ^{-1}(\mathrm{SD}=0.00055)$ is the release rate constant estimate for ${ }^{11} \mathrm{C}-(+)-\mathrm{PHNO}$ release from pancreas back to blood (amplitude $\mathrm{SD}=1.14 ; R=0.998$; data extracted with the WebPlotDigitizer 